\title{
ATS/ERS international multidisciplinary consensus classification of the idiopathic interstitial pneumonias
}

\author{
M. Demedts*, U. Costabel ${ }^{\#}$
}

In the January issue of the American Journal of Respiratory and Critical Care Medicine the "ATS/ERS international multidisciplinary consensus classification of idiopathic interstitial pneumonias" [1] was published, and this certainly deserves an editorial comment in the European Respiratory Journal (ERJ). Previously, several classifications have been proposed, of which the most influential were the landmark histological classification of chronic interstitial pneumonias by LIEBOW and CARRINGTON [2] and LiEBow [3], and the recent updates of acute and chronic idiopathic interstitial pneumonias (IIP) by Katzenstein and Myers [4] and by Müller and Colby [5].

A correct histological classification is of utmost importance based on the fact that prognosis and survival vary largely depending on the subset of IIP [6-8]. Pathology-based diagnoses are, however, only available in a minority of patients with IIP, since the majority do not undergo surgical lung biopsy. Conversely, it has been shown that high-resolution computed tomography (HRCT) scanning, in particular, but also other clinical features, may be of discriminative diagnostic value [5, 9-11]. Therefore, integrated clinical, radiological and histological classifications and definitions are mandatory. In response to this, the American Thoracic Society (ATS) and the European Respiratory Society (ERS) recently published an international consensus statement focusing on idiopathic pulmonary fibrosis (IPF), with guidelines on diagnosis and management of this most important subset of IIP, with its histological hallmark of usual interstitial pneumonia (UIP), as opposed to the other subsets of IIP [12].

The interest and dedication of the ERS in the development of the classification, diagnosis and management of IIP, and of interstitial lung diseases in general, is apparent from the fact that it recently published a monograph [13] and a supplement to the ERJ [14], updating these topics. The ERS was also involved in the ATS/ERS multidisciplinary consensus classification of IIP [1], which consequently means

\footnotetext{
*Division of Pneumology, University Hospital Gasthuisberg, Leuven, Belgium. " Dept Pneumology/Allergology, Ruhrlandklinik, Essen, Germany.

Correspondence: M. Demedts, Division of Pneumology, University Hospital Gasthuisberg, Herestraat 49, 3000 Leuven, Belgium. Fax: 32 16346803. E-mail: maurits.demedts@uz.kuleuven.ac.be
}

that results of previously published studies have to be updated to some extent.

The importance of the present ATS/ERS consensus classification is based on several issues. First, it provides an integrated clinical, radiological and pathological definition and classification of the whole group of IIP. Secondly, it proposes multidisciplinary consensus guidelines to establish uniform criteria for the diagnosis of the different IIP, aimed at pulmonologists as well as radiologists and pathologists. Third, while previous classifications have largely been conceived by individual authoritative pathologists or clinicians or by small collaborative groups of experts, the present consensus classification has been worked out by an international multidisciplinary core panel of some 20 pulmonologists, pulmonary pathologists and thoracic radiologists, and was then approved by an extended review panel of some 65 experts from over 20 countries. In addition, the document has been presented at the major clinical, radiology and pathology meetings over the past year, allowing for feedback from the practicing physicians involved in the care of patients with IIP.

The major new innovations in the document include: 1) providing a distinction between the diagnostic terminology of the disease entities e.g. IPF (which is based on the integration of clinical, radiological and pathological data) and the histological pattern e.g. UIP (table 1), thus solving the confusion, often present in the past, of mixing-up clinical and pathological terms. Indeed, IPF is a diagnostic entity that must have the UIP pattern, but the histological UIP pattern may also be found in other entities e.g. asbestosis, drug- or radiationinduced lung disease, collagen-vascular disease. 2) Rethinking the clinical entities and histological patterns of IIP and retaining the subsets, listed in table 1, in the order of relative frequency. 3) Defining the clinical, radiological and pathological characteristics of the various subsets of IIP and the differential diagnoses. 4) Listing the areas of uncertainty; this "state of the art" document not only discusses the current knowledge in this field, but also highlights the questions that still need answering and future studies that need to be performed.

The level of evidence for the statements and guidelines is largely that of expert opinion developed by consensus and well-conducted cohort studies, since publications on randomized-controlled trials are scarce. At least three important issues arise with 
Table 1.- Histological and clinical classification of idiopathic interstitial pneumonias"

\begin{tabular}{ll}
\hline Histological patterns & Clinical, Radiological, Pathological diagnoses \\
\hline Usual interstitial pneumonia & Idiopathic pulmonary fibrosis/cryptogenic fibrosing alveolitis \\
Nonspecific interstitial pneumonia & Nonspecific interstitial pneumonia (provisional) \\
Organizing pneumonia & Cryptogenic organizing pneumonia \\
Diffuse alveolar damage & Acute interstitial pneumonia \\
Respiratory bronchiolitis & Respiratory bronchiolitis interstitial lung disease \\
Desquamative interstitial pneumonia & Desquamative interstitial pneumonia \\
Lymphocytic interstitial pneumonia & Lymphocytic interstitial pneumonia \\
\hline
\end{tabular}

\#: unclassifiable interstitial pneumonia: some cases are unclassifiable for a variety of reasons; ${ }^{\top}$ : this group represents a heterogeneous group with poorly characterized clinical and radiological features that need further study; ${ }^{+}$: COP is the preferred term, but is synonymous with idiopathic bronchiolitis obliterans organizing pneumonia (BOOP).

the new classification. First, are there clinical, radiological or histological patterns which cannot be categorized into one of the retained entities i.e. is there still a group of "nonclassifiable" IIP or should all these be grouped in the nonspecific interstitial pneumonia (NSIP) subset, which thus becomes a "garbage bag?". The panel acknowledged the fact that there are a minority of cases which, for various reasons, remain unclassifiable after extensive clinical, radiological and/or pathological examination. The designation "unclassifiable interstitial pneumonia" should be used for, and limited to, such cases and not be applied to cases of clearly-defined NSIP or cases where the distinction between the UIP and fibrosing NSIP pattern is difficult. Second, what should the clinician do if surgical biopsies in different lobes of one lung show different histological patterns e.g. UIP, NSIP and organizing pneumonia? The paper, last year, by FLAHERTY et al. [15] gave a reply to this question by showing that $35 \%$ of patients with a histological pattern of UIP in any lobe, had NSIP in other lobes (so-called "discordant" UIP) and that they all had the same prognosis as concordant UIP and therefore, should be classified as UIP. The important consequence of this is that if in UIP the surgical biopsy is taken in only one lobe there is a $35 \%$ chance that it shows NSIP just by coincidence. Thus, in these conditions the HRCT showing UIP should prevail over histology! Third, one may question whether the term "organizing pneumonia" used for the histological correlation of the clinical entity "cryptogenic organizing pneumonia" (COP, also termed bronchiolitis obliterans organizing pneumonia (BOOP) in the American literature), is specific enough. Since the "majority of changes centre in small airways" (as stated in the document [1]) it appears more accurate to include this in the terminology e.g. by labelling the pathological definition as "bronchiolitis and organizing pneumonia".

Despite the points raised that still require some discussion, the value of this multidisciplinary consensus classification is enormous, even if it is not the last update on idiopathic interstitial pneumonias. It is the first truly worldwide international document on idiopathic interstitial pneumonias. All textbooks on respiratory medicine need to be rewritten to include this new classification. It is important that clinicians, pathologists and radiologists become accustomed to the new terminology and make proper use of it to benefit their patients with interstitial lung disease.

\section{References}

1. Travis WD, King TE, Bateman ED, et al. ATS/ERS international multidisciplinary consensus classification of idiopathic interstitial pneumonias. General principles and recommendations. Am J Respir Crit Care Med 2002; 165: 277-304.

2. Liebow AA, Carrington CB. The interstitial pneumonias. In: Simon M, Potchen EJ, LeMay M, eds. Frontiers of pulmonary radiology. New York, Grune and Stratton, 1969; pp. 102-141.

3. Liebow AA. Definition and classification of interstitial pneumonias in human pathology. Prog Respir Research 1975; 8: 1-33.

4. Katzenstein AL, Myers JL. Idiopathic pulmonary fibrosis: clinical relevance of pathological classification. Am J Respir Crit Care Med 1998; 157: 13011315.

5. Müller NL, Colby TV. Idiopathic interstitial pneumonias: high resolution CT and histologic findings. Radiographics 1997; 17: 1016-1022.

6. Bjoraker JA, Ryu JH, Edwin MK, et al. Prognostic significance of histopathologic subsets in idiopathic pulmonary fibrosis. Am J Respir Crit Care Med 1998; 157: 199-203.

7. Nagai S, Kitaichi M, Itoh H, Nishimura K, Izumi T, Colby TV. Idiopathic nonspecific interstitial pneumonia/fibrosis: comparison with idiopathic pulmonary fibrosis and BOOP. Eur Respir J 1998; 12: 10101019.

8. Travis WD, Matsui K, Moss IE, Ferrans VJ. Idiopathic nonspecific interstitial pneumonia: prognostic significance of cellular and fibrosing patterns. Survival comparison with usual interstitial pneumonia and desquamative interstitial pneumonia. Am J Surg Pathol 2000; 24: 19-38.

9. Raghu G, Mageto YN, Lockhart D, Schmidt RA, Wood DE, Godwin JD. The accuracy of the clinical diagnosis of new-onset idiopathic pulmonary fibrosis and other interstitial lung disease: A prospective study. Chest 1999; 116: 1168-1174.

10. Hunninghake GW, Zimmerman MB, Schwartz DA, et al. Utility of lung biopsy for the diagnosis of idiopathic pulmonary fibrosis. Am J Respir Crit Care Med 2001; 164: 193-196. 
11. Grenier P, Valeyre D, Cluzel P, Brauner MW, Lenoir S, Chastang C. Chronic diffuse interstitial lung disease: diagnostic value of chest radiography and high-resolution CT. Radiology 1991; 179: 123132.

12. King TE, Costabel U, Cordier J-F, et al. International Consensus Statement. Idiopathic pulmonary fibrosis: diagnosis and treatment. Am J Respir Crit Care Med 2000; 161: 646-664.
13. Olivieri D, du Bois RM. Interstitial lung diseases. Eur Respir Mon 2000; 14: 1-288.

14. Demedts M, du Bois RM, Nemery B, Verleden GM. Interstitial lung diseases: a clinical update. Eur Respir $J$ 2001; 18: Suppl. 32, 1s-133s.

15. Flaherty KR, Travis WD, Colby TV, et al. Histopathologic variability in usual and nonspecific interstitial pneumonias. Am J Respir Crit Care Med 2001; 164: 1722-1727. 